Exercices Exercices de rhétorique

de rhétorique $\quad 7 \mid 2016$

Les rhétoriques du peuple

De la difficulté de catégoriser le peuple (des invisibles) en échappant aux jugements de valeur

Alain Rabatel

CpenEdition

Journals

Édition électronique

URL : http://journals.openedition.org/rhetorique/457

DOI : $10.4000 /$ rhetorique.457

ISSN : 2270-6909

Éditeur

UGA Éditions/Université Grenoble Alpes

Édition imprimée

ISBN : 978-2-37747-084-6

Référence électronique

Alain Rabatel, « De la difficulté de catégoriser le peuple (des invisibles) en échappant aux jugements de valeur », Exercices de rhétorique [En ligne], 7 | 2016, mis en ligne le 28 mai 2016, consulté le 12 septembre 2020. URL : http://journals.openedition.org/rhetorique/457 ; DOI : https://doi.org/10.4000/ rhetorique.457

Ce document a été généré automatiquement le 12 septembre 2020.

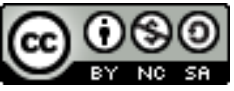

Les contenus de la revue Exercices de rhétorique sont mis à disposition selon les termes de la Licence Creative Commons Attribution - Pas d'Utilisation Commerciale - Partage dans les Mêmes Conditions 4.0 International. 


\title{
De la difficulté de catégoriser le peuple (des invisibles) en échappant aux jugements de valeur
}

\author{
Alain Rabatel
}

1 Sans entrer dans une longue discussion lexicologique sur les significations du mot peuple $^{1}$, il est indispensable de préciser le sens qui sera privilégié dans ce travail. À cette fin, je m'appuierai sur deux dictionnaires particulièrement représentatifs. Le mot peuple est défini dans le Grand Larousse de la Langue Française (GLLF) selon plusieurs niveaux: 1) l'ensemble d'individus formant une foule; 2) l'ensemble d'individus habitant le même lieu ; 3) l'ensemble d'individus constituant une nation définie par un territoire et des institutions politiques ${ }^{2}$; 4) la classe des gens qui vivent péniblement de leur travail, sens vieilli, mais qui renvoie aux couches/classes populaires, qui représenteraient la majorité, dont les intérêts seraient bafoués par un petit nombre de privilégiés, selon une représentation que d'aucuns jugent imaginaire, et d'autres, scientifique. Le Petit Robert (PR, édition 2014), distingue une signification ethnologique, culturelle, territoriale (le/s peuple/s $\mathrm{s}^{3}$ ); une signification politique-institutionnelle (le peuple corps de la nation, renvoyant à l'idée de souveraineté d'un tout pluriel mais indivisible); une signification sociale, concernant la masse ${ }^{4}$.

2 C'est cette dernière signification socio-politique (tant dans le GLLF que dans le $P R$ ) que je retiendrai pour analyser quelques représentations discursives du peuple, avec, en arrière-plan, les luttes de reconnaissance ${ }^{5}$ ou les procès en (il)légitimité qui accompagnent les discours sur le peuple ${ }^{6}$, selon la situation d'où l'on parle et le point de vue qu'on a sur lui. Il s'agit donc de discours sur le peuple - et non de discours (ou de paroles) $d u$ peuple, tels qu'ils sont reproduits dans des romans, par exemple chez Ernaux $^{7}$ ou dans des traités de sociologie, par exemple chez Bourdieu, dans La misère $d u$ monde. Évidemment, on peut encore faire des discours au nom du peuple. Cette fonction de porte-parole ne sera pas au cœur de mon propos. Mais il n'y a pas toujours une frontière nette entre parler sur le peuple et parler en son nom, dans la mesure où les discours sur le peuple sont parfois fortement dépendants des représentations ou des 
imaginaires que l'on se fait du peuple, surtout lorsqu'il est question de décrire un peuple «invisible », qui, à l'instar du tiers état, compte pour "rien » alors qu'il est « tout » et aspire à être « quelque chose ${ }^{8}$ ».

3 J'indique d'emblée que je traiterai de mises en discours du peuple (le mot et la chose) à partir de quelques stratégies de caractérisation d'entités globales complexes. Pour ce faire, j'évoquerai d'abord des façons de parler du peuple à partir de procédés de naturalisation ${ }^{9}$ qui présentent des conceptions en réalité subjectives et historicisées comme des données objectives, renvoyant à des essences transhistoriques, c'est pourquoi les énoncés ou discours contenant ces "purs absolus ${ }^{10}$ " peuvent être dits essentialisants. La naturalisation d'un point de vue relatif aux entités collectives va souvent de pair avec une caractérisation de l'objet du discours qui sélectionne des traits uniquement positifs (idéalisation) ou négatifs (diabolisation ${ }^{11}$ ). Cette généralisation de traits - valant pour l'entité et censément pour tous ses membres - repose sur des traits considérés comme caractéristiques, voire prototypiques (du point de vue de celui qui partage cette représentation), mais que ceux qui ne la partagent pas jugent stéréotypée. La thèse que je voudrais défendre est que, s'agissant d'une entité collective aussi complexe et lestée de valeurs que le peuple, il est difficile ${ }^{12}$ d'échapper aux catégorisations essentialisantes, idéalisantes ou diabolisantes et/ou difficile aussi de ne pas faire affleurer des jugements de valeurs, parce que parler du peuple, c'est toujours faire intervenir son rapport au peuple, sur fond de luttes de légitimité. C'est ce que j'essaierai de montrer en appuyant mon propos non sur un seul type de discours mais en observant des exemples de discours politiques (1.), de discours littéraires - autour de fragments romanesques des $\mathrm{XIX}^{\mathrm{e}}$ et $\mathrm{xx}^{\mathrm{e}}$ siècles idéalisant ou diabolisant les classes populaires - (2.), de discours scientifiques, avec des textes de sociologues ou de politologues analysant les notions de « culture populaire » et de « populisme » (3.).

\section{Des discours politiques de N. Sarkozy essentialisant le peuple par effacements énonciatifs, énoncés génériques et généralisants}

4 Le phénomène d'effacement énonciatif ${ }^{13}$ crée des effets d'évidence et de naturalisation, comme si la nomination apparemment transparente des référents renvoyait à une existence naturelle ou à une essence qui n'auraient pas à être (re)mises en question, ou, à tout le moins, soumises à la question. Ainsi dans les exemples (1) à (3), lors de la campagne des élections présidentielles de 2007, même si N. Sarkozy utilise fréquemment le je et les verbes de volonté, comme cela a été souvent remarqué, il combine son engagement avec l'effacement énonciatif pour l'arrière-plan qui sert de cadre à ses prédications, comme dans «Il faut cesser de faire du travailleur la seule variable d'ajustement de l'économie ». N. Sarkozy réactive les topoï du plèthos en se présentant comme le champion de la masse des victimes abandonnées et en transformant l'image (injuste) de l'okhlos par un discours valorisant (celui du laos) :

(1) Il faut cesser de faire du travailleur la seule variable d'ajustement de l'économie. Il faut cesser de faire du travailleur celui qui paye pour tous les autres. Je veux être le président de la réhabilitation des travailleurs et de la hausse du pouvoir d'achat. (Toulon, 7 février 2007)

(2) La gauche ne parle plus des travailleurs car elle ne défend plus que les statuts, elle oublie les travailleurs dont nous allons, nous, parler. Jaurès aimait les travailleurs, il 
les respectait... Les travailleurs n'arrivent plus à se loger... Les salaires trop bas ralentissent la croissance et démoralisent les travailleurs. (Toulouse, 12 mars 2007)

(3) Je suis venu vous parler de la culture ouvrière parce qu'il y a une façon d'être des ouvriers, un rapport particulier des ouvriers à la vie et au travail... Ici, à Besançon il y a une culture du travail, une culture ouvrière qui fut longtemps celle du textile et de l'horlogerie qui a été durement frappée par la crise. Cette culture n'est pas morte, il ne faut pas qu'elle meure. (Besançon, 13 mars 2007

Ces phénomènes passent notamment par des impersonnalisations (« il faut », en (1), « il y a », en (3)), des infinitivations ou des passivations, des nominalisations (à l'instar de la " réhabilitation », en (1)), des GN ou des GV à valeur générique. Si dans certains genres de discours l'effacement évite autant que faire se peut l'emploi de subjectivèmes, comme dans le discours scientifique, il n'en va pas de même lorsqu'il y a polémique, comme dans les discours électoraux où l'on déforme les positions de l'adversaire. Le résultat est que la prédication, avec ses subjectivèmes, se donne comme exprimant un point de vue certain, indiscutable ${ }^{15}$, partagé par tous, dans lequel la subjectivité de l'énonciateur n'a plus de part réelle, sauf à consonner avec la vérité générale : c'est ce qui se passe en (2) : « Les salaires trop bas ralentissent la croissance et démoralisent les travailleurs ». Ainsi certains énoncés avec effacement se donnent-ils comme totalement objectivants («les travailleurs n'arrivent plus à se loger», en (2)), d'autres comprennent des subjectivèmes dont le degré négatif ("trop bas ») sert à exprimer une loi quasi objective, proportionnelle, entre baisse des salaires et baisse de la croissance et du moral.

6 Ces effets sont renforcés lorsque l'effacement énonciatif se cumule avec des énoncés à dimension générique et généralisante ${ }^{16}$, qui donnent au point de vue (particulier) défendu une apparence de vérité générale, avec, dans les exemples précédents, le singulier catégorisant "du travailleur», de "La gauche » (comme s'il n'en existait qu'une), ou l'emploi générique de l'indéfini (« une culture ouvrière »). Certes, il arrive à N. Sarkozy d'employer le pluriel (« des travailleurs»), mais c'est pour les envisager comme un tout, ainsi que le confirment les expansions de «la culture ouvrière »: " parce qu'il y a une façon d'être des ouvriers, un rapport particulier des ouvriers à la vie et au travail». C'est pourquoi, dans les citations précédentes, les syntagmes pourraient être précédés par l'un ou l'autre déterminant («la/une culture ouvrière », « une/la façon d'être des ouvriers ») puisque ces derniers expriment la même vision globale essentialisante des substantifs ainsi mis en discours. Comme Bourdieu ${ }^{17}$ l'écrivait, le peuple est une catégorie « à géométrie variable » dont, selon les intérêts du moment, on peut étendre la catégorie aux petits patrons et/ou aux paysans, ou la restreindre aux ouvriers de l'industrie, comme c'est le cas ici. Les ouvriers évoqués par N. Sarkozy sont «tout le peuple», vu les destinataires et vu surtout l'objectif affiché : car il s'agit de montrer que LE peuple (souffrant, exploité, qui se pense en principe à Gauche) est trahi par la Gauche, d'où la captation de l'héritage jaurésien, critiquée par les historiens ${ }^{18}$. Cette façon de présenter «les travailleurs» comme un tout emblématique - le petit peuple (dèmos) par rapport aux puissants - abstraction faite des différences, essentialise et idéalise la classe ouvrière (mais N. Sarkozy n'utilise bien évidemment pas ce terme, il préfère parler « des travailleurs », terme plus neutre, aux contours plus larges) pour gagner leurs voix.

7 Mais ces exemples de discours politiques de mauvaise foi - leur auteur lui-même parle de propos « limite mauvaise foi ", voire «terrifiant[s] de mauvaise foi ${ }^{19}$ " - ne sont pas représentatifs de tous les discours politiques. Certes, des propos de meetings électoraux 
ne prédisposent pas à la nuance et il est fréquent de se positionner dans son camp en étant le plus fougueux à pourfendre le contre-discours de l'adversaire, quitte à le déformer. Ce genre de pratiques n'est pas généralisable à tout discours politique, d'autant qu'il n'y a pas un discours politique, mais beaucoup de sous-genres distincts (discours de politique générale, débat, intervention en commission parlementaire, rapport, texte de loi, profession de foi, programme, etc.).

En outre, toute généralisation essentialisante n'est pas par nature mensongère. Les discours pédagogiques ou scientifiques sont remplis de telles tournures génériques ou généralisantes. Le discours essentialisant n'est pas manipulateur et réducteur par nature, il permet des approximations, des généralisations qui peuvent être utiles, comme le rappelle Frédéric François :

(4) C'est un peu comme quand on dit après un vote: "Les Français ont décidé que... » ou « La France a décidé que... ». Nous savons bien que ce n'est pas un vrai ensemble. Faut-il ici se contenter du mécanisme linguistique ordinaire d'adaptation $\mathrm{du}$ sens des mots les uns aux autres? Ou revenir sur le fait que ce «collectif» a, quand même, une réalité à travers l'institution? Ou encore rappeler que le discours générique n'est qu'un moment d'un discours (ou d'une pratique) ? Et le discours peut préciser ensuite, par exemple : «Mais le niveau des abstentions est de plus en plus élevé.» Il ne s'agit pas tant de vérité ou d'erreur que de niveau d'approximation qui semble suffisant ou non à tel récepteur ${ }^{20}$.

C'est donc moins la façon de dire qui fait problème que sa réitération, sa systématicité, ses visées, surtout lorsque ces façons de parler visent à figer, instrumentaliser les identités, comme dans les exemples ci-dessus. Cependant, ces instrumentalisations du peuple ou de ses fractions (partisans ou adversaires) sont loin de se limiter aux polémiques électorales, elles se rencontrent dans d'autres circonstances conflictuelles, par exemple dans le discours littéraire, lorsque les écrivains mettent en scène des figures du peuple systématiquement idéalisées ou rabaissées, en fonction de valeurs antagonistes.

\section{Deux imaginaires littéraires stéréotypés idéalisant ou diabolisant le peuple par une posture systématique de surplomb}

Les discours littéraires évoquent rarement le peuple. Lorsqu'ils le font, surtout s'ils parlent du peuple avec une posture de surplomb, il est fréquent que les représentations s'appuient sur des représentations prototypiques voire stéréotypées, comme dans les romans à thèse ${ }^{21}$. Les personnages populaires sont souvent traités comme des représentants typiques de leur classe, leurs traits physiques étant associés à des valeurs morales ou politiques exemplaires. Dans les romans qui relèvent du Proletkult ou du Jdanovisme, les ouvriers, vigoureux, au regard direct et franc, sont des révolutionnaires. $\mathrm{Du}$ prototype au stéréotype et aux imaginaires ${ }^{22}$, il n'y a pas loin... Ainsi, dans Le Donbass, Boris Gorbatov raconte l'histoire de deux jeunes héros komsomols, André Voronko et Victor Abrassimov, qui luttent pour les nouvelles méthodes de production, le stakhanovisme, vers 1930. Le narrateur n'y va pas par quatre chemins, en concluant ainsi le premier chapitre qui vient de présenter André et Victor, évoquant une jeunesse saine, qui a un bon fond natif, qui ne demande qu'à penser plus juste, grâce à l'aide 
d'éclaireurs avisés, selon des stéréotypes massifs qui occupent tout l'espace de la représentation :

(5) C'étaient de braves gars, simples et honnêtes, avec des yeux avides d'impressions, des idées vagues et généreuses, des rêves flous et agités, une âme largement ouverte au bien; je tiens donc beaucoup à ce que vous les aimiez comme je les aime ${ }^{23}$.

Quant aux héros, qui veulent abattre des masses considérables de charbon pour construire l'État soviétique, leur but n'est pas seulement d'augmenter la production et les rendements, il est de produire des hommes nouveaux : "le vainqueur ne sera pas celui qui abattra le plus de charbon, mais celui qui formera le plus d'élèves ${ }^{24}$ », dit un des compétiteurs. Et de même que les héros positifs sont d'un bloc, ils sont présentés en osmose totale avec l'État/le parti, comme le montre le discours direct de «l'ouvrier » :

(6) Quel était leur mobile ? Voilà ce qu'il devait expliquer à lui-même et à la réunion du Parti. Qu'est-ce qui les poussait ? L'appât du gain et des honneurs, l'ambition, la vanité, l'impatience, le sentiment du devoir ? Quel intérêt primait chez eux, le leur propre ou celui de la communauté ? Certes, les deux se confondaient. Et c'était là le secret du succès de la méthode stakhanoviste. Rien de plus clair pour l'ouvrier. «Je m'en trouve bien, et l'État aussi !» comme l'avait dit hier à la relève l'abatteur Soukhobokov, d'ordinaire si taciturne.

Que c'était bien dit! Quelle formule laconique et expressive pour un propagandiste ! Cependant, Soukhobokov n'était ni propagandiste, ni membre du Parti. Pourquoi? se demanda subitement Nétchaenko. Pourquoi n'est-il pas communiste? Et Mitia Zakorko, pourquoi n'a-t-il toujours pas adhéré au Parti? Il allait donc rester komsomol toute sa vie ? Et Otchérétine ? Et Zakorliouka-aîné ?

Bouleversé, Nétchaenko s'était levé et arpentait la pièce. « C'est donc que je ne vaux rien comme partorg! Il faut le dire franchement à la réunion. Dans chaque homme, si insociable, si arriéré qu'il soit, couve l'étincelle de l'activité sociale, il suffit de savoir l'attiser. Et c'est à nous, les communistes, de le faire, à moi en premier lieu ${ }^{25} . »$

12 Il n'y a pas de différence, chez Nétchaenko, entre le discours de la délibération intérieure et le discours public au comité du «Parti »- il n'y en a qu'un et il mérite sa majuscule. Et de même, les intérêts des travailleurs sont ceux du parti : en chacun d'eux préexiste une " étincelle » qui doit être attisée pour que les individus fassent un, dans le Parti. Si les personnages ne sont pas assez explicites, le narrateur y pourvoit, en (7), en évoquant une fusion, une communion, avec intériorisation volontariste de l'impératif moral/politique. La participation à la réunion requiert l'abandon des « petites affaires privées », « insignifiantes et mesquines »:

(7) Vous avez certainement constaté qu'en se rendant à une réunion du Parti, on se sent toujours très ému, comme si c'était la première fois. On éprouve le besoin de se nettoyer, de se rectifier, de mettre de l'ordre en soi. Vos petits problèmes quotidiens reculent au dernier plan: les petites affaires privées deviennent tout à fait insignifiantes et mesquines en comparaison de la grande cause commune pour laquelle on va se réunir. [...]

Aux réunions, le général voisine avec le soldat, l'ajusteur côtoie le ministre : ils sont tous membre du même Parti ${ }^{26}$.

13 Le narrateur a un point de vue surplombant, monologique dont la visée est de souligner la communauté de vues et d'intérêts des personnages et de l'État, avec son organisation d'avant-garde, le Parti, ses satellites, les Komsomols : la représentation des individus ne laisse aucune place à des différences individuelles, sauf pour les asociaux antipartis sur lesquels pèsent bien des menaces qui se lisent en filigrane par contraste avec cette idéalisation-fusion au cœur du processus d'essentialisation ${ }^{27}$. 
14 Si la fusion va de pair avec les représentations idéalisantes du peuple, il n'en va bien évidemment pas de même dans les représentations diabolisantes, mais au total, l'idéalisation comme la diabolisation s'appuient sur une position de surplomb qui ne s'attache qu'aux traits jugés essentiels, typiques, appelant un jugement moral qui ne peut pas ne pas être partagé. C'est le cas des portraits à charge du peuple chez des écrivains hostiles au monde ouvrier, comme Léon Bloy ${ }^{28}$. Ainsi, dans La Femme pauvre, le romancier catholique, dont la plume pamphlétaire n'est jamais loin, décrit-il les ouvriers et ce qui les touche (leur logement, par exemple), en multipliant les hyperboles et les sarcasmes violemment critiques. Nulle charité chrétienne dans cette description de l'ouvrier, du peuple, il s'agit d'une description d'un point de vue surplombant (celui de qui possède la vérité de la foi), sans connivence avec le peuple, victime de l'ironie et du sarcasme du narrateur ${ }^{29}$.

(8) Le tabernacle était sinistre, éclairé par le livide plafond de ce ciel glacé de fin d'automne. Mais on peut supposer que le soleil rutilant des Indes l'aurait fait paraître encore plus horrible.

C'était la noire misère parisienne attifée de son mensonge, l'odieux bric-à-brac d'une ancienne aisance d'ouvriers bourgeois lentement démeublée par la noce et les fringales.

D'abord, un grand lit napoléonien qui avait pu être beau en 1810, mais dont les cuivres dédorés depuis les Cent Jours, le vernis absent, les roulettes percluses, les pieds eux-mêmes lamentablement rapiécés et les éraflures sans nombre attestaient la décrépitude. Cette couche sans délices, à peine garnie d'un matelas équivoque et d'une paire de draps sales insuffisamment dissimulés par une courtepointe gélatineuse, avait dû crever sous elle trois générations de déménageurs.

Dans l'ombre de ce monument, qui remplissait le tiers de la mansarde, s'apercevait un autre matelas, moucheté par les punaises et noir de crasse, étalé simplement sur le carreau. De l'autre côté, un vieux voltaire, qu'on pouvait croire échappé au sac d'une ville, laissait émigrer ses entrailles de varech et le fil de fer, malgré l'hypocrisie presque touchante d'une loque de tapisserie d'enfant. [...]

Tel était le mobilier, assez semblable à beaucoup d'autres dans cette joyeuse capitale de la bamboche et du désarroi.

Mais ce qu'il y avait de particulier et d'atroce, c'était la prétention de dignité fière et de distinction bourgeoise que la compagne sentimentale de Chapuis avait répandue, comme une pommade, sur la moisissure de cet effroyable taudis ${ }^{30}$.

15 En effet, tous les traits descriptifs exemplifient « la noire misère parisienne attifée de son mensonge », et toutes les figures - hyperboles négatives (" avait dû crever sous elle trois générations de déménageurs»); ironie ("ce monument», "distinction bourgeoise »); métaphores (" courtepointe gélatineuse »), etc. - intensifient la charge négative elle-même fortement exprimée à travers des termes dont le sémantisme évoque la négativité avec une intensité maximale ("horrible», «infâme», "effroyable»). La description hyperbolique sarcastique est au service d'une visée disqualifiante de l'esthétique, la sociologie et la vision politique du plèthos et de l'okhlos, comme le confirme la dimension métonymique du mobilier délabré, signe du délabrement moral des habitants, eux-mêmes étant le symbole du peuple selon le positionnement politique et esthétique des naturalistes, du moins aux yeux de leurs adversaires catholiques de droite, tel Bloy.

Cela étant, de même que les discours politiques sur le peuple ne sont pas tous essentialisants, les discours littéraires sur ce dernier ne versent pas tous dans une diabolisation ou une idéalisation essentialisantes, car les figures du peuple ne se réduisent pas aux seuls Nétchaenko ou Chapuis, représentants d'un peuple fantasmé, 
comme le montre la représentation empathique, proche et cependant distanciée, des voix populaires chez Annie Ernaux ${ }^{31}$.

\section{Catégoriser sans essentialiser et sans jugements de valeur? Les exemples de la « culture populaire » et du " populisme » dans les sciences sociales}

Mutadis mutandis, les discours scientifiques éprouvent aussi des difficultés à parler du peuple ou de catégories affines. Si l'approche essentialisante est en principe bannie, elle rejaillit parfois derrière des analyses catégorisantes qui peuvent conduire à des lectures essentialisantes, indépendamment de la visée de l'auteur (exemple (9)) ou comprendre des jugements de valeur personnels alors que leur auteur met à distance ceux des autres (exemple (10)).

(9) Tout se passe comme si l'« esthétique populaire était fondée sur l'affirmation de la continuité de l'art et de la vie, qui implique la subordination de la forme à la fonction, ou, si l'on veut, sur le refus du refus qui est au principe même de l'esthétique savante, c'est-à-dire la coupure tranchée entre les dispositions ordinaires et la disposition proprement esthétique. L'hostilité des classes populaires et des fractions les moins riches en capital culturel des classes moyennes à l'égard de toute espèce de recherche formelle s'affirme aussi bien en matière de théâtre qu'en matière de peinture ou, plus nettement encore parce que la légitimité en est moindre, en matière de photographie ou de cinéma. Au théâtre comme au cinéma, le public populaire se plait aux intrigues logiquement et chronologiquement orientées vers une happy end et se «retrouve » mieux dans les situations et les personnages simplement dessinés que dans les figures et les actions ambiguës et symboliques ou les problèmes énigmatiques du théâtre selon Le théâtre et son double, sans même parler de l'existence inexistante des "héros" pitoyables à la Beckett ou des conversations bizarrement banales ou imperturbablement absurdes à la Pinter $^{32}$.

Chez Bourdieu, la culture populaire est présentée comme un bloc de pratiques représentant une vision du monde homogène. S'agit-il d'une catégorisation essentialisante? Ailleurs, Bourdieu la rapporte à une histoire socialisée, sujette à des évolutions. Mais ici, Bourdieu présente son analyse dans le cadre d'une fiction, comme l'indique le «tout se passe comme si » initial. Certes, cette fiction est la marque d'un travail de reconstruction. Néanmoins, elle fait parler une esthétique populaire bien évidemment incapable de parler - pas plus d'ailleurs que le public ne parle d'une seule voix - et elle y associe le lecteur, un lecteur qui parle comme Bourdieu : «l'affirmation de la continuité de l'art et de la vie, qui implique la subordination de la forme à la fonction, ou, si l'on veut, [...] le refus du refus qui est au cœur même de l'esthétique savante ». Le texte avance sans discuter son principe fondamental («l'affirmation de la continuité de l'art et de la vie» a une valeur axiomatique) ni ses conséquences ( "implique »). L'alternative libérale consentie au lecteur (" ou si l'on veut ») n'est guère qu'une reformulation bourdieusienne au suprême degré d'une définition de la culture populaire par distinction d'avec «l'esthétique savante». Bref, la façon dont Bourdieu catégorise cette culture est globalement homogénéisante, peu spécifiante, du fait de l'opposition structurale à la culture savante. Et, malgré son auteur, cette conception systémique peut nourrir des interprétations essentialisantes en raison de traits que l'interprétation structurale tend à déshistoriciser. L'on peut comprendre que, dès lors, d'aucuns lui aient opposé une analyse plus diversifiée des réseaux, parcours, effectués 
par des acteurs, et non par des agents (Lahire, Latour ${ }^{33}$ ). D'un autre côté, on peut relativiser cette critique en invoquant la visée structurale, systémique, qui oppose culture savante et culture populaire, sans compter les nombreuses enquêtes de terrain, ou encore les précautions de Bourdieu ${ }^{34}$ lorsqu'il évoque la catégorie de "populaire ", ou revient sur elle. Quoi qu'il en soit, il y a là une écriture complexe, montrant que la tentative de catégorisation peut prêter à des analyses linguistiques et sociologiques diverses. Ces manières d'écrire ne sont sans doute pas les seules à justifier certaines critiques de Bourdieu - par exemple Lahire reproche de trop figer la catégorie et de sous-estimer les parcours des acteurs ${ }^{35}$ - mais sans doute les ont-elles alimentées. Il existe néanmoins une différence nette avec les exemples précédents: la visée descriptive ne repose pas sur une quelconque instrumentation idéalisant ou diabolisant le peuple. Néanmoins, (9) témoigne d'une difficulté à dire et sans doute à circonscrire la catégorie, comme pour la notion de peuple.

On rencontre des problèmes analogues avec une notion qui n'a certes rien à voir avec la culture populaire, mais beaucoup avec celle de peuple, à savoir la notion très discutée de populisme, qui concerne/conteste une certaine conception de la politique dans son rapport au peuple. On objectera qu'il n'y a rien de commun entre une analyse des pratiques (ci-dessus) et un retour réflexif sur des usages discursifs (ci-dessous). Mais c'est bien cela qui nous interroge, à savoir le fait que des notions différentes, mais néanmoins affines, en ce qu'elles tentent de circonscrire la constellation de notions associées à l'entité collective "peuple ", soient toutes difficiles à dire. Assurément, on pourrait dire de même de beaucoup de termes renvoyant à d'autres entités collectives, mais le "peuple » est particulièrement touché par ce phénomène, en raison de forts enjeux politiques, éthiques, voire esthétiques. C'est ce que montre bien l'exemple (10), qui traite de la notion de populisme en interrogeant le caractère hautement désémantisé et donc la plasticité sémantique de la mention sous ses avatars populaires, non sans échapper aux jugements de valeur.

(10) Il est arrivé une singulière mésaventure au mot «populisme»: il est récemment devenu populaire. Le terme étant sorti du langage savant, « ses usages dominants » s'inscrivent désormais dans l'espace polémique occupé par les acteurs politiques, les journalistes et les intellectuels médiatiques. On sait ce qu'il est advenu de l'adjectif « surréaliste », lorsque ses usages se sont étendus et banalisés («C'est surréaliste!») au point de l'évider de tout sens précis. Cette désémantisation est le prix à payer pour toute extension indéfinie d'une catégorie d'usage courant ("C'est baroque!», "C'est décadent!», «C'est raciste!»). Le suremploi à toutes fins utiles prive un mot de sa pertinence, le rend non seulement inutile, mais aussi dangereux, voire nuisible. N'irait-on pas jusqu'à soutenir, non sans quelque raison, qu'user d'un mot dégradé est un acte dégradant? Quoi qu'il en soit, la réduction récurrente d'un mot à un instrument de blâme ou d'éloge en rend fort difficile les éventuels usages conceptuels, comme si un pli rhétorique avait été pris une fois pour toutes. Les usages récents du mot "populisme » semblent avoir pris le même pli que ceux du mot "fascisme» dans le langage ordinaire: le suremploi polémique a fait de ce terme une étiquette disqualificatoire et un opérateur d'amalgame permettant de stigmatiser, en les rassemblant abusivement, un certain nombre de phénomènes sociopolitiques ou de leaders jugés détestables ou redoutables par celui qui les dénonce. Significativement, l'épithète " populiste » n'apparaît guère que dans des expressions polémiques: "dérive populiste", " tentation populiste », « danger (ou menace) populiste », voire " prurit populiste », et le «populisme» est dénoncé comme l'incarnation du «mal européen», le principal facteur de division et de conflit en Europe. 
Ainsi contextualisée, cette catégorie péjorante qui réfère à une sourde menace semble avoir été reprise telle quelle, dans un premier temps, au sein des milieux savants: l'examen critique de la notion n'a pas précédé l'usage du terme. Aux États-Unis dans les années 1950, le maccarthysme était dénoncé comme une forme de « populisme » tandis qu'en France on croyait assister, dans la «fièvre électorale» du poujadisme (1953-1956), à une mobilisation " populiste ", qui reparaîtra en Grande Bretagne, à la fin des années 1960 et au début des années 1970, avec le powellisme ${ }^{36}$.

21 Après le commentaire sur la désémantisation de la mention, la notion, dans ses usages, est contextualisée sous des formes différentes, et renvoyée à une visée stigmatisante qui confirme selon l'historien que la notion est plus souvent une arme de délégitimation politique qu'une catégorie scientifique. Cela dit, ce moment est le prélude à une approche savante, qui seule semble rendre possible une approche scientifique non instrumentalisée de la notion. Le mouvement est paradoxal, car tout en pourfendant l'usage péjoratif d'une "catégorie péjorante », Rioux reproduit un tel jugement de valeur en affirmant que la notion s'est perdue en devenant " populaire » et de facto polémique, comme si les approches scientifiques, au demeurant, échappaient au conflit! Autrement dit, tout se passe comme si parler des fractions basses du dèmos était si difficile que le discours stigmatise ceux qui veulent parler au nom du plèthos. Comme dans les extraits de Bourdieu, il est malaisé de parler du peuple directement ou à travers des notions affines, d'objectiver des entités globales complexes. Néanmoins les exemples (11) et (12) s'y essaient, sous la forme d'une typologisation, d'une approche historicisée qui abandonne les jugements de valeur dévalorisants :

(11) La multiplicité et l'hétérogénéité des critères retenus pour définir le " populisme » viennent de ce qu'ils relèvent de différents domaines de signification. On peut en distinguer au moins six. 1/ le populisme-mouvement; [...] 2/ le populisme-régime $[. ..] ; 3 /$ le populisme-idéologie $[. .$.$] ; 4/ le populisme-attitude [...] ;$ 5/ le populisme-rhétorique [...] ; 6/ le populisme-type de légitimation ${ }^{37}$ [...].

(12) Une intéressante tentative de clarification a été faite par Margaret Canovan, dans son livre paru en 1981, Populism. Pour mettre de l'ordre dans l'indéfinie diversité des populismes, celle-ci présente une suggestive typologie des phénomènes populistes, fondée sur une première distinction entre deux grandes catégories : le populisme agraire et le populisme politique. Le populisme agraire est de trois types ${ }^{38}$.

L'exemple (11) déploie cette typologie dans les pages 36 à 41 en évoquant un certain nombre de sous-types qui sont autant de facettes d'un phénomène complexe, disparate, aux plans historique, géographique, idéologique. En (12), la typologie est rapportée à son auteure, en sorte que, le circuit de la construction n'étant pas omis ${ }^{39}$, la tentation de la naturalisation est écartée. D'autant plus que la typologie est présentée non comme un absolu, mais comme une "tentative de clarification »- présupposant des analyses antérieures opaques. Celle-ci est qualifiée d' «intéressante ", de " suggestive ", caractéristiques jugées pertinentes pour penser et classer une réalité complexe. C'est pourquoi «le populisme", avec des guillemets de mise à distance d'emploi unifiant, unique, essentialisant, en (10), est remplacé en (12) par l'indéfini pluriel «des populismes ». La typologie est présentée comme un essai de mise en ordre, rapporté à des opérations intellectuelles (typologie, catégories) qui renvoient certes à des formes types, mais la matière est d'une part découpée par des sujets, d'autre part historicisée, c'est pourquoi, selon les critères retenus, les typologies varient. 

affines) tient à la posture d'extériorité extralinguistique ou linguistique, d'autant que cette posture surplombante s'accommode tantôt de son idéalisation (Gorbatov), tantôt de sa diabolisation (Bloy). Si la position de surplomb scientifique ne s'accompagne pas des instrumentations exemplifiées dans des discours politiques ou littéraires sur le peuple, elle n'échappe cependant pas, sous une forme ou sous une autre, aux jugements de valeur, surtout lorsque les questions portent sur une fraction dominée du peuple, qui aspire à la reconnaissance. Même si le sociologue ou l'historien ne s'érigent pas en porte-paroles du peuple, quand ils écrivent sur le peuple, ils le pensent à partir de valeurs ambivalentes, fortement intriquées, comme le rappellent les relations entre dèmos, laos, plèthos et ethnos. Cette intrication est encore plus problématique si on la réfléchit en termes de (légitimité de) place, de pouvoir des uns et des autres, questions auxquelles n'échappent pas les auteurs qui écrivent sur le peuple.

\section{NOTES}

1. Et plus encore sur ses rapports avec des termes connexes renvoyant eux aussi à des entités collectives complexes, état, nation, pays, patrie, république, voir S. Remi-Giraud \& P. Retat dir., Les Mots de la nation, Lyon, Presses universitaires de Lyon, 1996.

2. C'est le dèmos, habitant le territoire du dème (ibid., p. 19). Mais les relations entre la conception territoriale du peuple et sa définition institutionnelle (les citoyens) sont complexes - comme le montre la comparaison avec la formulation de la deuxième signification du Petit Robert. C'est encore plus vrai si on pense que le dèmos peut soit renvoyer à l'ensemble des citoyens, soit au petit peuple par rapport aux puissants (plousios).

3. C'est l'ethnos, qui évoque la nation ou la race (ibid., p. 20).

4. On retrouve là - comme dans l'acception 1) du GLLF - le plèthos grec, évoquant la foule, la multitude : voir M. Grodent, « De dèmos à populus », Hermès, 42, 2005, p. 19-22 ; p. 20.

5. Voir A. Honneth, La Lutte pour la reconnaissance, Paris, Éditions du Cerf, [1992] 2000.

6. Souvent ces luttes opèrent à l'intérieur d'un même peuple, par exemple à travers les conflits avec ses dirigeants ou des luttes de classes. Mais elles ont lieu aussi entre peuples : c'est ce dernier niveau qui est privilégié par les Verts français, dont Cavalla analyse le concept de peuple, à partir de l'emploi exclusif du mot à propos de l'opposition «peuples du Sud »vs «peuples du Nord", dichotomie qui légitime leur combat pour un ordre mondial plus équitable. (C. Cavalla, «Les "peuples" dans le programme des Verts », dans H. Desbrousses, B. Peloille \& G. Raulet dir., Le Peuple, figures et concepts : entre identité et souveraineté, Paris, François Xavier Guibert, 2003, p. 276-289).

7. Voir A. Rabatel, «La Re-présentation des voix populaires dans le discours auctorial chez A. Ernaux : surénonciation et antihumanisme théorique ", dans A. Petitjean \& J.-M. Privat dir., Effets de voix populaires dans les fictions romanesques et théâtrales, Recherches textuelles, 7, Université de Metz, 2007, p. 287-325.

8. On se rappelle les premiers mots de l'ouvrage de Sieyès : " $1^{\circ}$ Qu'est-ce que le Tiers état ? TOUT. $2^{\circ}$ Qu'a-t-il été jusqu'à présent ? - RIEN. $3^{\circ}$ Que demande-t-il ? - À ÊTRE QUELQUE CHOSE » (Emmanuel Joseph Sieyès, Qu'est-ce que le Tiers état ?, Paris, Éditions du Boucher, [1789] 2002, p. 1). 
9. Voir J.-M. Klinkenberg, Périphériques Nord, Liège, Les Éditions de l'Université de Liège, 2010, p. 57-58.

10. Ibid.

11. Ces représentations sont déjà au cœur de la langue et de la culture grecques : le laos évoque le peuple de façon poétique valorisante, l'okhlos (populace) est dépréciatif (M. Grodent, « De dèmos à populus », op. cit., p. 20).

12. Difficile mais pas impossible, on pourrait en alléguer de nombreux exemples. Cependant mon propos n'est pas là.

13. Voir P. Charaudeau, Grammaire du sens et de l'expression, Hachette, Paris, 1992 ; R. Vion, " "Effacement énonciatif" et stratégies discursives», M. De Mattia \& A. Joly dir., De la syntaxe à la narratologie énonciative, Paris - Gap, Ophrys, 2001, p. 331-354; A. Rabatel, Homo narrans. Pour une analyse énonciative et interactionnelle du récit. Tome 1. Les points de vue et la logique de la narration. Tome 2. Dialogisme et polyphonie dans le récit, Limoges, Éditions Lambert-Lucas, 2008, p. 577-580.

14. Dans J.-L. Calvet \& J. Véronis, Les Mots de Nicolas Sarkozy, Paris, Le Seuil, 2008, p. 122-123. Nous soulignons.

15. Voir également la valeur énonciative du car, en (2), qui présente le jugement initial accusant la gauche comme une évidence partagée par tous.

16. Tout énoncé générique ( "l'homme est un loup pour l'homme », « on a toujours besoin d'un plus petit que soi ») a une dimension généralisante, mais celle-ci est plus ou moins forte selon le degré de stéréotypie des SN ou des SV : ainsi un énoncé tel que « le travailleur est celui qui paye pour tous les autres » est plus généralisant que les exemples ci-dessus.

17. P. Bourdieu, "Vous avez dit 'populaire' ?", Actes de la recherche en sciences sociales, 46, 1983, p. $98-105 ;$ p. 98.

18. L. De Cock, F. Madeline, N. Offenstadt \& S. Wahnich dir., Comment Nicolas Sarkozy écrit l'histoire de France, Marseille, Agone, 2008.

19. J.-L. Calvet \& J. Véronis, op. cit., p. 150.

20. F. François, Bakhtine tout nu. Ou Une lecture de Bakhtine en dialogue avec Vološinov, Medvedev et Vygostki, ou encore Dialogisme, les malheurs d'un concept quand il devient trop gros, mais dialogisme quand même, Limoges, Lambert-Lucas, 2012, p. 148.

21. S. R. Suleiman, Le Roman à thèse ou l'autorité fictive, Paris, Presses universitaires de France, 1983.

22. A.-M. Houdebine-Gravaud dir., L'Imaginaire linguistique, Paris, L'Harmattan, 2003 ; P. Charaudeau, «Les stéréotypes, c'est bien, les imaginaires, c'est mieux », dans H. Boyer dir., Stéréotypage, stéréotypes: fonctionnements ordinaires et mises en scène, Paris, L'Harmattan, 2007, p. 49-63.

23. B. Gorbatov, Le Donbass, Moscou, Éditions En Langues Étrangères, 1953, p. 24.

24. Ibid., p. 257.

25. Ibid., p. 423-424.

26. Ibid., p. 37-39.

27. Le contexte du Jdanovisme n'est pas celui du Proletkult, l'idéalisation n'a pas le même sens à l'époque héroïque de la construction du socialisme et à celle du stalinisme finissant, après une énième période de glaciation...

28. Là encore, genre et contexte devraient être soigneusement pris en compte : pour des analyses plus détaillées, voir J.-M. Seillan, Le Roman idéaliste français dans le second XIX siècle. Littérature ou 'bouillon de veau' ?, Paris, Classiques Garnier, 2011.

29. Pour une analyse plus complète, voir A. Rabatel, «Donner à voir le visible? La vision opacifiante de Bloy dans La Femme pauvre ", dans A. de Georges-Métral dir., Poétiques du descriptif dans le roman français du XIX ${ }^{e}$ siècle, Paris, Classiques Garnier, 2014.

30. Léon Bloy, La Femme pauvre : épisode contemporain, Paris, G. Crès, [1897] 1924, p. 13-15. 
31. Voir A. Rabatel, «La re-présentation des voix populaires dans le discours auctorial chez A. Ernaux : surénonciation et antihumanisme théorique », op. cit.; A. Rabatel, « La fictionnalisation des paroles et des gestes dans Les Années d'Annie Ernaux », Poétique, 173, 2013, p. 105-123.

32. P. Bourdieu, La Distinction, Paris, Éditions de Minuit, 1979, p. 33-34.

33. Voir A. Rabatel, «La dialectique du singulier et du social dans l'approche énonciative du style à travers l'articulation des primats et des primautés, des facteurs et des acteurs ", dans D. Ablali \& M. Kastberg dir., Littérature et linguistique, Cluny, 40 ans après. Textes : normes, usages et pratiques, Besançon, Presses universitaires de Franche-Comté, 2010, p. 325-336.

34. P. Bourdieu, « Vous avez dit 'populaire'? », op. cit.

35. Voir A. Rabatel, « La dialectique du singulier et du social dans l'approche énonciative du style à travers l'articulation des primats et des primautés, des facteurs et des acteurs », op. cit.

36. J.-P. Rioux dir., Vingtième siècle. Revue d'histoire, 56, [nº́cial], «Les populismes », Paris, Éditions Perrin, coll. Tempus, [1997] 2007, p. 17-18.

37. A. Taguieff, «Le Populisme et la science politique », dans J.-P. Rioux dir., op. cit., p. 17-59; p. 31-36.

38. Ibid., p. 39.

39. Voir B.-N. Grunig, "Pour une conception dynamique du sujet», dans M. Yaguello dir., Subjecthood and Subjectivity. The status of the subject in linguistic theory, Paris - Gap, Ophrys, 1994, p. $125-137$; p. 129.

\section{AUTEUR}

\section{ALAIN RABATEL}

Université de Lyon 1 